\title{
Common Immune Mediated Skin Disease of Dog
}

\author{
Fentahun W Molla ${ }^{1 *}$, Temesigen W Molla ${ }^{2}$, Dagimai Yitibarek ${ }^{2}$, Yitayew Demisie ${ }^{2}$ and Kibebe Legese ${ }^{2}$ \\ ${ }^{1}$ Department of Veterinary Medicine, Addis Ababa University, Ethiopia \\ ${ }^{2}$ Department of Veterinary Medicine, University of Gondar, Ethiopia
}

Submission: October 4, 2016; Published: November 15, 2016

*Corresponding author: Fentahun W Molla, Department of Veterinary Medicine, Adis Ababa University, Adis Ababa, Ethiopia,

Tel: +251 910388 030; Email: bebehalf.31@gmail.com/temu3544@gmail.com

\section{Summary}

All organisms, even trees, have what could be considered an innate defense system. For trees it would be their bark, for dogs it would be their skin and for a bacterium it would be the cellular wall around it. The immune system protects the host from infection by bacterial, fungal and viral pathogens. To accomplish this there is a complex system of humeral and cellular immune reactants that interacts to provide this protection. The basic premise for protective immunity is the ability to differentiate self from non-self. The Major Histocompatibility Complex (MHC) plays a crucial role in the vertebrate immune system by encoding a collection of immune and non-immune related molecules.

When this fails immune mediated disease takes place in the normal system. Among such diseases autoimmune dermatologic/ immune mediated diseases represent some of the most interesting and challenging problems seen in veterinary dermatology. For instance pemphigus foliaceus (PF) and discoid lupus erythematosus (DLE) are frequently occurring immune mediated skin diseases. The exact immunopathogenesis of this group of diseases is unknown but the production of antigen-antibody complexes and subsequently lodge in small vessels and the basement membrane zone of the skin for DLE and involvement of either abnormal immune regulation or abnormal antigen stimulation to PF is noted.

The initial lesions in DLE are an area of depigmentation or erythema that slowly progresses to loss of the normal cobblestone appearance of the nasal planum and eventual development of erosions, ulcers and crusts and scaring in severe and chronic cases. While in PF lesions consist of erythematous macules that progress rapidly to a pustular phase and then appear as a dry, yellow crust which is limited to the pinnal, perioral, periocular and dorsal muzzle, nasalplanm and nail bed regions. These ailments are diagnosed by cytology of pustules, laboratory testing (CBC, serum biochemistry profile and ANA) and histopathology of primary skin lesions and IF and immunoperoxidase testing. To treat DLE; applying topical glucocorticoids to the planum nasale, tetracycline and niacin amide in combination and by use of azathioprine in severe cases indicated. Likewise for PF we can use prednisone as a single therapeutic agent and azathioprine or with chlorambucil, tetracycline and niacin amide, cyclospor-ine, tacrolimus (topical), Dapsone, sulfasalazine or aurothiomalate. The prognosis for both of these ailments is fair to good. If glucocorticoids cannot be administered, then the prognosis is Poor to guard.

Keywords: Immune system; MHC; Immune mediated diseases; PF; DLE

Abbreviations: MHC: Major Histocompatibility Complex; DLE: Discoid Lupus Erythomathosis; SLE: Systemic Lupus Erythomasosis; ECLE: Exfoliative Cutaneous Lupus Lrythematosus; VCLE: Vesicular Cutaneous Lupus Erythematosus; UV: Ultra Violet; ANA: Antinuclear Antibody; IFA: Immuno Florescent Assay; CIMDA: Canine Immune Mediated Disease Awareness; AIHA: Autoimmune Hemolytic Anemia; PF: Pemphigus Fallacious; CBC: Complete Blood Count; PO: Peros; IF: Immuno Florescence

\section{Introduction}

The immune system is the surveillance and defense system of the body. It recognizes foreign substances by their molecular features and eliminates them from the body. It is an organization of cells and molecules with specialized roles in defending against infection. The immune system can be divided into two parts based on how specific their functions are. It has two divisions called the innate immune system and the adaptive immune system [1].

The body can potentially respond to almost anything that can be bound by the receptors of either the innate or the acquired immune system. Molecules recognized by receptors 
on lymphocytes are generically referred to as antigens and can range from small chemical structures to highly complex molecules. Both the T-cell receptor and the antibody that is embedded in the B-cell membrane, the B-cell receptor, have binding sites 1, 2 that are only 600 to $1700 \AA$ A. Therefore, these receptors recognize only a small part of a complex antigen, referred to as the antigenic epitope.

Not all antigens are naturally immunogenic. Small, nonimmunogenic antigens are called haptens and must be coupled to larger immunogenic molecules, termed carriers, to stimulate a response. Carbohydrates must often be coupled to proteins in order to be immunogenic, as is the case for the polysaccharide antigens used in the haemophilus influenza type B vaccine [1].

The immune system protects the host from infection by bacterial, fungal and viral pathogens. To accomplish this there is a complex system of humeral and cellular immune reactants that interacts to provide this protection. The basic premise for protective immunity is the ability to differentiate self from non-self. This ability is acquired during fetal development. The primary lymphoid organ thymus "educates" fetal thymic lymphocytes so that those that enters the periphery and become mature [2].

Recognition of self from non-self involves interaction of naive immature thymic lymphocytes with thymic epithelial cells. These cells express a wide variety of tissue antigens as well as major histocompatibility antigens II (MHC) and I. The immature T cells are "tested" for their ability to bind to self-MHC antigens. Those that do not bind at all are subject to induction of apoptosis and are eliminated. Those that bind too strongly are similarly disposed of. The T cells with ability to recognize MHC of self, but not to bind strongly enough to elicit a cytotoxic event are retained [3].

When the above mechanism fails immune mediated disease takes place in the normal system. Among such diseases autoimmune dermatologic/immune mediated diseases represent some of the most interesting and challenging problems seen in veterinary dermatology. For instance pemphigus foliaceus (PF) and discoid lupus erythematosus (DLE) are frequently occurring immune mediated skin diseases [4].

In DLE initial lesion is an area of depigmentation or erythema that slowly progresses to loss of the normal cobblestone appearance of the nasal planum and eventual development of erosions, ulcers and crusts. Scarring may occur in severe and chronic cases. The planum nasale is the area most commonly affected, although lesions have been noted on the eyelids, lips, foot pads and concave surface of the pinnae and in the oral cavity. Profuse hemorrhage may occur following minor trauma to the planum nasale. Sunlight exacerbates the lesions and may play a role in the pathogenesis of DLE [5].
$\mathrm{PF}$ is the most common form pemphigus complex which may be either spontaneous or drug induced especially with trimethoprim sulfas. Lesions consist of erythematous macules that progress rapidly to a pustular phase and then appear as a dry, yellow crust. These lesions may be limited to the pinnal, perioral, periocular, dorsal muzzle, nasalplanm and/or nail bed regions or they may be generalized [6].

To treat DLE; applying topical glucocorticoids to the planum nasale, tetracycline and niacin amide in combination and by use of azathioprine in severe cases indicated. Likewise for PF we can use prednisone as a single therapeutic agent and azathioprine or with chlorambucil, tetracycline and niacinamide, cyclosporine, tacrolimus (topical), Dapsone, Dapsone, sulfasalazine or aurothiomalate [7].

To this end the objectives of this manuscript are

A. To inform the adverse effect of skin immune mediated disease on our companion animals, especially dogs both in their life and comfort.

B. To recall veterinarian that drugs are more likely to kill the patient than the disease unless appropriately indicated.

\section{Immune mediated disorders}

Autoimmune dermatologic diseases represent some of the most interesting and challenging problems seen in veterinary dermatology. The immune system protects the host from infection by bacterial, fungal and viral pathogens. To accomplish this there is a complex system of humeral and cellular immune reactants that interacts to provide this protection. The basic premise for protective immunity is the ability to differentiate self from non-self. This ability is acquired during fetal development. The primary lymphoid organ thymus "educates" fetal thymic lymphocytes so that those that enters the periphery and become mature [2].

$\mathrm{T}$ lymphocytes that do not react in an adverse way with host cells and tissues, but are able to assist in the elimination of pathogens and other foreign cells that enter the host. Nonetheless there are situations in which an immune response may be generated such that self-tissues are attacked. These responses are referred to as "autoimmune" and depending upon which of the self-antigens.

The immune response is directed towards; clinical signs of disease occur and are relevant to the functions of those target tissues/organs. One common example is autoimmune hemolytic anemia. In autoimmune hemolytic anemia antibodies bind specifically with antigenic epitopes on self-erythrocytes causing loss of red blood cells and subsequent anemia. Recognition of self from non-self involves interaction of naive immature thymic lymphocytes with thymic epithelial cells. These cells express a wide variety of tissue antigens as well as major histocompatibility antigens II (MHC) and I. 
The immature T cells are "tested" for their ability to bind to self-MHC antigens. Those that do not bind at all are subject to induction of apoptosis and are eliminated. Those that bind too strongly are similarly disposed of. The $\mathrm{T}$ cells with ability to recognize MHC of self, but not to bind strongly enough to elicit a cytotoxic event are retained. These cells become either CD4 or CD $8 \mathrm{~T}$ cells and can bind to MHC class II or MHC class I, respectively, while their $\mathrm{T}$ cell receptor (TCR) for antigen has specificity of some foreign epitope. The TCR's are screened for reactivity to the promiscuously expressed tissue antigens on thymicepithelial cells and those that react with any of these antigens are induced into apoptosis and eliminated from the $\mathrm{T}$ cell pool that will enter the periphery to seed the secondary lymphoid organs [3].

The majorities of B cells become tolerant and therefore do not respond to self-antigens in an adverse way, the screening of these cells is less rigorous than for $\mathrm{T}$ lymphocytes. Thus, there are B cells present in the body that are capable of recognizing and binding to some self-epitopes. However, the lack of T cells reactive with those antigens keep the $\mathrm{B}$ cells in check as they require $\mathrm{T}$ cell help in order to initiate an immune response and antibody production. One well recognized mechanism occurs when the target tissue is in a privileged site, such that the $\mathrm{T}$ and B cells were never exposed to its tissue specific antigens during development. These sites include CNS tissues, the lens of the eye and sperm forming cells in the testicle. If a traumatic event exposes these tissues to the adult immune system an immune attack on the organ or tissue is a common sequel.

Another well recognized mechanism occurs when there are shared antigen epitopes between a host tissue and a pathogen, such as a virus or bacteria. The presence of helper $\mathrm{T}$ cells specific for the pathogen makes it possible for B cells that are not tolerized to the cross-reactive antigens, to use those $\mathrm{T}$ cells to establish the co-stimulatory signals required for activation and differentiation into antibody producing plasma cells. The resultant antibodies can then attack the self- tissues and evoke inflammation and tissue destruction [2].

\section{Common skin immune mediated disease in dogs}

Lupus erythematosus complex: This includes discoid lupus erythematosus (DLE), systemic lupus erythematosus (SLE), exfoliativecutaneouslupus erythematosus (ECLE) and vesicular cutaneous lupuserythematous (VCLE) [4].

Pemphigus Complex: The pemphigus complex of diseases includes pemphigus foliaceus, pemphigus erythematosus, pemphigus vulgaris, pemphigus vegetans, panepidermal pustular pemphigus, Paraneoplastic pemphigus and drug related pemphigus. The pemphigus complex includes four subtypes recogn-ized in human medicine, pemphigus vulgaris, vegetans, erythematosus and foliaceus. In all of these subtypes, the immune system for various and often unknown reasons starts to produce antibodies against parts of the desmosomes, the little connections holding adjacent keratinocytes together. These (auto) antibodies are called pemphigus antibodies. The antibodies in the different subtypes are directed against different antigens of the desmosomes expressed in different layers of the epidermis.

Thus, the location of the forming vesicles within the epidermis and the resultant clinical signs vary. Once the antibody is bound to the part of the desmosome (specialized structure of the cell membrane especially of an epithelial cell that serves as a zone of adhesion to anchor contiguous cells together which forms the antigen, the complex is "swallowed" by the cell and elicits intracellular reactions leading to the release of plasminogen activator. The subsequent plasminogen activation results in the production of plasmin, a protease that destroys the desmosomes and leads to acantholysis (the process, where keratinocytes lose their intercellular bridges and "round up"). These acantholytic cells are located as single cells in vesicles formed by the destruction of the intercellular connections [8].

Pemphigus foliaceus is the most common autoimmune skin disorder in dogs and cats, while pemphigus erythematosus is considered a variant of pemphigus foliaceus. It may have clinical and histopathologic features of lupus erythematosus and is therefore considered a "crossover" between the pemphigus and the lupus erythematosus complexes. On the other hand pemphigus vegetans is an extremely rare variant of pemphigus vulgaris that is distinguished clinically from the other autoimmune diseases by the production of lesions that are vegetative (i.e., proliferative) rather than pustular or ulcerative [4].

\section{Predisposing Factors}

Genetic factors: About $60 \%$ of dogs is now purebred and pure breeding always involves in breeding. Because of the great diversity of immune mechanisms and hence the genetic complexity of its development and regulation, it is understandable how inbreeding can result in a spectrum of disorders ranging from dysregulation of the immune system and autoimmunity at one extreme to cancers at the other. Although it may be over simplistic, the susceptibility to autoimmune diseases is thought to be controlled by the interplay of environmental and heritable factors; the latter involving genes of the major histocompatibility complex (MHC) [9].

Sex: The gender of the dog is the second most common cofactor intact females have the highest incidence of immune diseases and intact males the lowest, as has been observed in humans. The gender bias is not nearly as obvious with wide spread spaying and castration. Ovariohysterectoy reduces the incidence, while castration increases the incidence, thus tending to equalize gender effects in neutered animals [10]. 
The presence of other immunologic disorders: The third most common factor underlying immune diseases is the presence of other immunologic disorders. If a certain breed or bloodline of dogs suffers from one immune disease, they will also have an increased incidence of a wide range of other immunologic disorders, as demonstrated in a study of the old English sheepdog. Plasmacytic/lymphocytic thyroiditis and hypothyroidism is a common occurrence among dogs that develop other types of immune diseases. Dogs with systemic lupus erythematosus may also present with autoantibody associated cytopenias [11].

Infectious diseases: The fourth most common cofactor is infectious diseases. Infections can trigger allergies, the formation of autoantibodies immune complex diseases, cell mediated pathologies, gammopathies or immune deficiencies [12].

Drugs: The fifth most common factor underlying immunologic diseases in dogs is drugs. Drugs, including biologics, especially when given to dogs with certain genetic predispositions, can trigger a variety of immune reactions [13].

\section{Clinical features}

\section{Lupus erythematosus complex}

Etiology: The exact immunopathogenesis of this group of diseases is unknown. Antigen-antibody complexes are produced and subsequently lodge in small vessels and the basement membrane zone of the skin (SLE and DLE) and in various organ systems (SLE). Genetic factors, Tcell defects, B cell hyperactivity, hormonal alterations and viral inducement of antigen-antibody complex formation has all been implicated [14].

Genetic factors are important and experimental colonies of dogs with SLE have been established. SLE patients produce antibodies directed against a broad range of nuclear, cytoplasmic and cell membrane molecules. Auto antibodies may directly damage tissues or cause damage through the formation of immune complexes. When there is continued production of autoantibody to a self-antigen, overload of the mononuclear phagocyte system may occur. Circulating immune complexes will deposit in walls of blood vessels where there is physiological outflow of fluid, such as glomeruli, synovia and the choroid plexus [15].

Pathogenesis: The exact pathogenesis of DLE is not elucidated in dogs and cats. However, a subset of dogs with clinical signs definitely deteriorates when exposed to UV light and improves on UV protection, thus it is safe to assume that UV rays play a role in at least some of the patients with DLE. Another subset of animals does not seem to be influenced at all by changes in UV exposure. Breed predispositions exist for Collies, Shetland sheepdogs, German shorthaired pointers, Siberian Huskies and Brittany Spaniels. Thus, genetic factors seem to play a role in the disease. Histopathologically, there is a spectrum of changes as well, but plasma cells are more prominent in dogs than humans (where lymphocytes are the major inflammatory cells) [14].

\section{Clinical sign}

Discoid lupus erythematosus: Discoid lupus erythematosus (DLE) is one of the more frequently encountered autoimmune skin diseases in dogs and humans. In humans, $10 \%$ of all patients with DLE proceed to develop systemic lupus erythematosus (SLE). Luckily, this does not seem to happen in small animals, only rarely will a patient with DLE develop systemic signs [14].

There are no internal manifestations of disease except in very rare "potential" crossover cases that, in time, become more consistent with SLE. The initial lesion is an area of depigmentation or erythema that slowly progresses to loss of the normal cobblestone appearance of the nasal planum and eventual development of erosions, ulcers and crusts. Scarring may occur in severe and chronic cases. The planum nasale is the area most commonly affected, although lesions have been noted on the eyelids, lips, foot pads and concave surface of the pinnae and in the oral cavity. Profuse hemorrhage may occur following minor trauma to the planum nasale. Sunlight exacerbates the lesions and may play a role in the pathogenesis of DLE [5].

Systemic lupus erythematosus: Collies and Shetland sheepdogs are predisposed. SLE is a multiorgan disease with dermatologic manifestations in $32 \%$ to $54 \%$ of cases. Cutaneous signs associated with SLE include ulcerative stomatitis, seborrhea mucocutaneous ulceration, foot pad ulceration, panniculitis, urticaria and purpura. Noncutaneous signs of SLE include polyarthritis, fever, glomerulonephritis, hemolytic anemia, thrombocytopenia, polymyositis, neurologic signs, pleuritis, myocarditis and lymphade-nopathy [15].

Exfoliative cutaneous lupus erythematosus: This hereditary disease (autosomal recessive) is also known as lupoid dermatosis of Germanshorthairedpointers. It usually occurs in the first year of life. It is characterized by localized to generalized scale with follicular casting and alopecia. Pruritus is minimal. The course may wax and wane. Dogs may also have blood dyscrasias, joint disease and features of sebaceous denitis [15].

Vesicular cutaneous lupus erythematosus: This is also known as ulcerative dermatosis of collies and Shetland sheepdogs and was previously thought to be a variant of dermatomyositis. It has an adult onset. Lesions are annular, polycyclic and serpiginous ulcerations and are located primarily in the inguinal and axillary regions [15].

\section{Diagnosis}

Discoid lupus erythematosus: ANA test is positive in $5 \%$ of cases of DLE. This may indicate those animals with the potential for conversion to SLE that therefore should be closely monitored. Histopathologic findings include a lichenoid interface dermatitis composed primarily of lymphocytes and plasma cells, 
thickened basement membrane zone, hydropic degeneration of the basal cell layer, apoptotic keratinocytes in the lower layers of the epidermis, pigmentary incontinence and excess of dermal mucin. IFA and IPS are positive, with a granular or rough band of immunoglobulin and/or complement deposited at the basement membrane zone [14].

Systemic lupus erythematosus: Direct smears are nondiagnostic. $\mathrm{CBC}$, serum biochemical profile and urinalysis may show a variety of abnormalities, depending on the non-cutaneous organs involved. ANA test is positive in $85 \%$ to $90 \%$ of cases. The negative titers in $10 \%$ to $15 \%$ of ANA tests maybe a laboratory "fault" because of the current inability of most laboratories to test for extractable nuclear antigens [14].

The lupus erythematosus cell preparation is unreliable and not routinely used in veterinary medicine. Histopathologic findings include epidermal and dermal lesions similar to those seen in DLE and may include leukocyt oclastic vasculitis and mononuclear panniculitis. IFA and IPS are positive, with a band of staining at the basement membrane zone of the skin and involved dermal and subcutaneous vessels [5].

Exfoliative cutaneous lupus erythematosus: Histopathology reveals hyperkeratosis, basal cell degeneration and apoptotic epidermal cells, as well as features of sebaceous adenitis [15].

Vesicular cutaneous lupus erythematosus: Histopathology reveals an interface dermatitis and folliculitis composed predominantly of lymphocytes with vesiculation of the dermalepidermal junction [15].

Differential diagnosis: Other poly articular diseases affecting dogs, like rheumatoid arthritis undifferentiated connective tissue disease, primary anti phospholipid syndrome, idiopathic thrombocytopenic purpura, drug induced lupus and autoimmune thyroid disease are the most common one(Crawford , 1989). Patients presenting with fever or splenomegaly/ lymphadenopathy also mimic this disease. In febrile patients with known SLE, leucocytosis, neutrophilia, shaking chills and normal levels of anti DNA antibodies favor infection. Lupus may present with localized or generalized lymphade-nopathy or splenomegaly, but the size of lymph nodes is rarely $>2 \mathrm{~cm}$ while splenomegaly is mild-to-moderate. Patients with known or suspected SLE with prominent lymphadenopathy, massive splenomegaly or expansion of a monoclonal B cell population should raise the suspicion of non-Hodgkin lymphoma [5] (Figure 1).
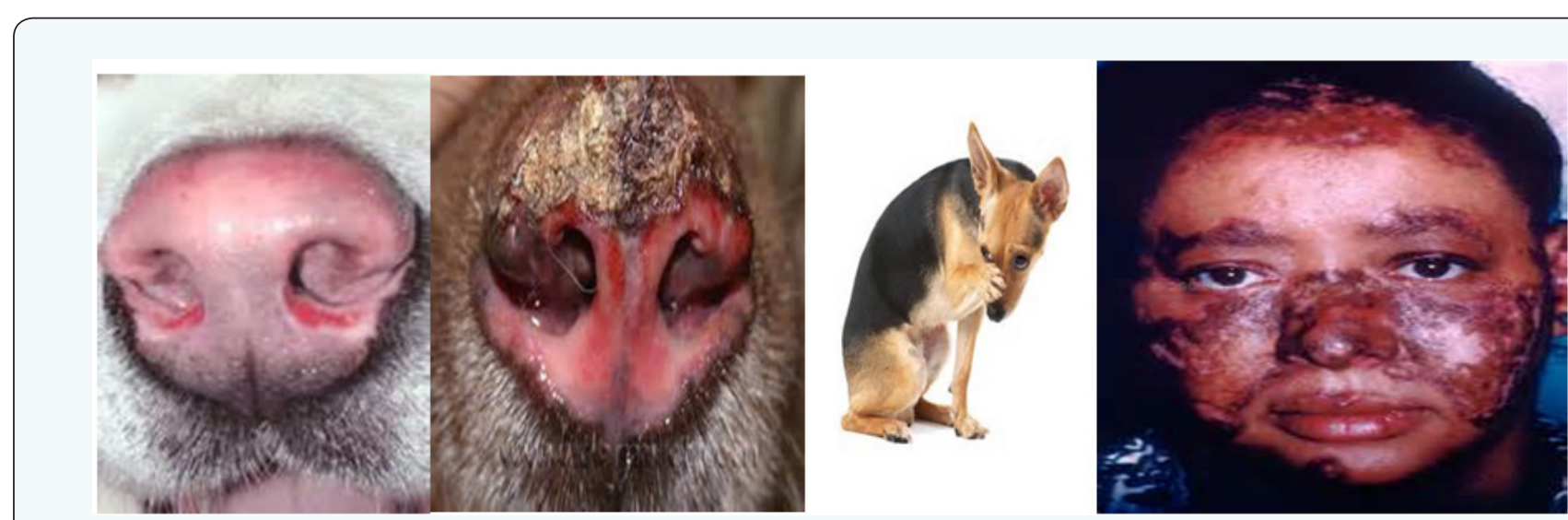

Figure 1: Clinical Sign of DLE [20].

\section{Treatment of lupus erythematosus complex}

To treat SLE, give prednisone, $1 \mathrm{mg} / \mathrm{kg}$ PO q12h.Systemic glucocorticoids are rarely necessary in DLE; thus, only use them in severe DLE that is unresponsive to other therapies. Apply topical glucocorticoids to the planum nasale. This may be the only form of corticosteroid needed to treat and control mild cases [7]. Topical tacrolimus (Protopic $0.03 \%$ or $0.1 \%$ ) may be beneficial Apply topical sunscreens to the planum nasale during periods of sun exposure. Administer vitamin E 400 IU PO q12h. Give 2 hours before or after a meal. Vitamin E has a 30 to 60 day lag phase and therapy usually is maintained for the life of the $\operatorname{dog}[7]$.

Tetracycline and niacinamide in combination may be effective. In dogs over $10 \mathrm{~kg}$, the initial dosage is $500 \mathrm{mg}$ of each drug PO q8h and in dogs under10 $\mathrm{kg}$, the dosage is 250 $\mathrm{mg}$ of each drug PO q8h.Once response is noted, the dosage can be decreased to $\mathrm{q} 12 \mathrm{~h}$ and then to $\mathrm{q} 24 \mathrm{~h}$. Side effects include anorexia, vomiting and diarrhea [7].

Severe cases may require the use of azathioprine. Gold salts are contraindicated in SLE because of the potential for both the drug and the disease to produce glomerulonephritis. Essential fatty acid supplementation may be used as an adjunct therapy for all of the disorders [7].

\section{Pemphigus complex}

\section{Etiology}

The exact cause or stimulant for the production of the pemphigus antibody is unknown. Theories involve either 
abnormal immune regulation or abnormal antigen stimulation. A virus spread by an insect vector has been proposed as the initial stimulus. This theory gains support froman endemic form of pemphigus in humans in South America. Genetic factors may be equally important as there are breed predilections for the disease [16].

In humans, once formed, the antibody binds with components found in the core of the desmosome. Desmosomes function as attachment areas between keratinocytes of the skin. This binding stimulates plasminogen activators (i.e., serine proteases), which subsequently cause the conversion of plasminogen to plasmin. The production of plasmin causes the disruption of the desmosome attachments and therefore a loss of keratinocyte adhesion. This loss of adhesion between adjacent cells is called acantholysis and the individual cells are termed acantholytic cells [17].

All of the diseases in the pemphigus complex appear to have the same immunopathogenesis, but the target protein will vary depending on the type of pemphigus. The location of the bulla or separation within the epidermis differs; for example, pemphigus foliaceus has a more superficial bulla than pemphigus vulgaris [16].

\section{Pathogenesis}

Lesions develop from antibodies binding antigen resulting in intracellular adhesion breakdown between keratinocytes with different layers of the epidermis. This results in cell separation (acantholysis) and blister formation [6].

\section{Clinical sign}

Pemphigus Foliaceus: It has no sex predilection but bread predisposition occurs. It is the most common form which may be either spontaneous or drug induced especially with trimethoprim-sulfas. Lesions consist of erythematous macules that progress rapidly to a pustular phase and then appear as a dry, yellow crust. These lesions may be limited to the pinnal, perioral, periocular, dorsal muzzle, nasalplanm and/or nail bed regions or they may be generalized [6] (Figure 2).

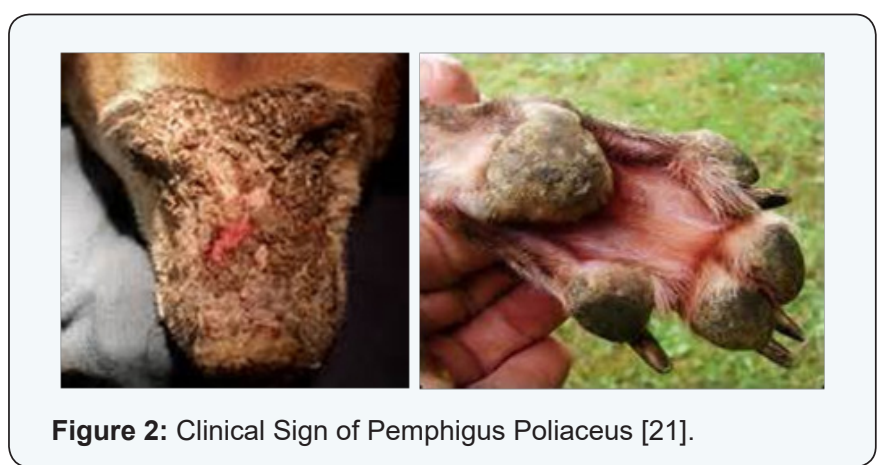

Although pustules are the primary lesions, these are uncommonly seen and the clinician is more typically presented with a crusting dermatitis. Animals may present with marked hyperkeratosis (scaling) or crusting of the foot pads with or without nail bed involvement. The nails usually are normal [16].

Pemphigus Erythematosus: Pemphigus erythematosus is a rarely recognized variant of pemphigus foliaceus but mild form. Lesions are similar to those of pemphigus foliaceus but are limited to the face. Some animals may show depigmentation of the planum nasale [14].

Pemphigus vulgaris: It is the second rarest but most severe form of pemphigus. Lesions are characterized as vesicobullous eruptions that rapidly ulcerate, leaving thick crusts. Lesions may be primarily mucocutaneous in location or generalized. Onychomades is (loss or shedding of the nails) and foot pad ulcerations are common. Ulceration of the oral cavity may be an initial presenting sign in over $50 \%$ of cases and is eventually present in $90 \%$ of cases. Drooling and difficulty in eating may be presenting signs. Pruritus and pain are variable [18].

Pemphigus vegetans: Pemphigus vegetans is an extremely rare variant of pemphigus vulgaris so called benign variant of. Lesions usually are generalized rather than mucocutaneous. Lesions are vegetative (proliferative) or verrucous (wart-like) [18].

Pan epidermal pustular pemphigus: Lesions are limited to the face. Pustules are found at all levels within the epidermis and follicular epithelium. Lesions are short-lived pustules that rupture and form a thick, adherent crust [5].

Drug-related pemphigus: Pemphigus may be drug induced or drug triggered. The drug-induced form often resolves when the medication is withdrawn, while the drug-triggered form usually requires immunosuppressive therapy. Trimethoprimsulfa is the most common drug associated with pemphigus. Feline cases are associated with itraconazole, methimazole and lime sulfur dips [15].

\section{Diagnosis}

Pemphigus complex can be diagnosed by cytology of pustules, laboratory testing $(\mathrm{CBC}$, serum biochemistry profile and ANA) and histopathology of primary skin lesions (pustules, bulaes and vesicles) and IF and immunoperoxidase testing [6].

Histopathologic examination of skin biopsies gives the most valuable diagnostic information. Obtain at least three biopsies of the freshest pustules, vesicles or bullae or from the edge of an ulcerated lesion. In all of the pemphigus complex diseases, the complete blood count ( $\mathrm{CBC}$ ), serum biochemical profile and urinalysis are non-diagnostic. ANA tests are positive at low titers in $50 \%$ of pemphigus erythematosus patients, but in other forms of pemphigus the ANA tests are generally negative.

False-positive ANA titers may be seen in animals with pemphigus, rheumatoid arthritis, idiopathic thrombocytopenia, autoimmune hemolytic anemia, thyroiditis, endocarditis, cancer, hepatotoxicity, feline leukemia, feline infectious peritonitis, 
dirofilariasis, demodicosis and flea allergy dermatitis; they even may be seen in clinically normal animals [5].

\section{Pemphigus foliaceus}

Direct smear of an intact pustule or surface beneath a thick crust reveals numerous acantholytic cells. Histopathology findings include subcorneal and/or intragranular pustules with acantholytic cells [5].

Direct immunofluorescent antibody (IFA) tests and directimmunoperoxidase staining (IPS) tests are positive, with intercellular staining of immunoglobulin and/or complement in the upper one-third of the epidermis. IFA is positive in only $50 \%$ of autoimmune cases, whereas IPS is very sensitive andis positive in $95 \%$ of confirmed cases. IPS is not very specific; positive results are obtained in $73 \%$ of animals with pyoderma, $67 \%$ with dermatophytes, $50 \%$ with demodicosis and $100 \%$ with scabies. Also, IPS is positive with the immunoreactant immunoglobulin $\mathrm{G}$ in an intercellular pattern in biopsies obtained from normal canine planum nasale and foot pads. IFA is positive with the immunoreactant immunoglobulin $\mathrm{M}$ in a basement membrane zone pattern in $75 \%$ of normal canine nasal biopsies and $45 \%$ of biopsies of normal foot pads, thus yielding false-positive results [5].

\section{Pemphigus erythematosus}

Direct smears are similar to those of pemphigus foliaceus. ANA tests are positive, with low titers, in $50 \%$ of cases. Histopathologic findings include subcorneal and/or intragranular pustules, hydropic degeneration of the basal cell layer and dyskeratotic cells. IFA and IPS are positive with staining in the intercellular pattern with or without concurrent staining in the basement membrane zone [18].

Pemphigus Vulgaris: Direct smears are similar to those of pemphigus foliaceus. Histopathologic findings include suprabasilar pustules with acantholysis. IFA and IPS are positive, with intercellular deposition of immunoglobulin or complement in the lower one-third of the epidermis [18].

\section{Pemphigus vegetans}

Direct smears are similar to those of pemphigus foliaceus. Histopathologic findings include intraepidermal acantholytic eosinophilic microabscesses with significant surface crusting and verrucous vegetations and papillomatous proliferations. IFA and IPS are positive, with an intercellular staining pattern [15].

\section{Pan epidermal pustular pemphigus}

Histopathology findings include sterile pustules involving the subgranular layers of the epidermis and outer root sheath of the hair follicle, suppurative crusts with acantholytic cells, irregular epidermal hyperplasia and a superficial perivascular and interstitial inflammatory response [15].

\section{Differential Diagnosis}

The major differential diagnoses for pemphigus foliaceus include the following

\section{Generalized pustular crusting}

Bacterialpyoderma, dermatophytosis, demodicosis, cutaneous drug reaction, dermatophilosis, subcorneal pustulardermatoses, sterile eosinophilic pustulosis are the most frequently occurring cases [19].

\section{Pedal lesions}

Superficial necrolytic dermatitis, systemic lupus erythematosus/ discoid lupus erythematosus, zinc-responsive dermatoses and contact dermatitis and digital hyperkeratosis are the common ones [18].

\section{Nasal or facial lesions}

Bacterial pyoderma, dermatophytosis, demodicosis, cutaneous drug reaction, dermatomyositis, pemphigus erythematosus and discoid lupus erythematosus are the most confusing cases [18].

\section{Treatments}

The goal in treating autoimmune skin diseases is to keep the condition in satisfactory remission on a "safe" dose of medication. It is better for the animal to have a few remaining lesions on low-dose alternate-day glucocorticoids than to have normal skin on high daily doses of glucocorticoids. All in all we can use prednisone as a single therapeutic agent with azathioprine or with chlorambucil, tetracycline and niacinamide, cyclosporine, tacrolimus (topical), Dapsone, Dapsone sulfasalazine or aurothiomalate (gold salts) to treat your skin immune mediated disease affected dog [7].

For initial therapy, choose prednisone. If the response is poor, add a chemotherapeutic agent such as azathioprine to the protocol. For more rapid and complete resolution of clinical signs with less resistance to therapy, you shall start with a regimen of prednisone and an adjunctive chemotherapeutic drug. In treating these disorders maintaining medications at a high dose until clinical signs have resolved at least $75 \%$ to $85 \%$, then gradually decrease dosages while monitoring for exacerbation of disease is recommended. Taper either the prednisone or the chemotherapeutic agent first, depending on side effects noted. Another option is to alternate the dosage reductions of the two medications until there is complete remission and therapy is no longer necessary or until the minimum dosage of drug needed to control the disease is found [7].

\section{Prognosis}

The prognosis for control of pemphigus foliaceus is fair to good. If glucocorticoids cannot be administered, then the prognosis is poor to guarded. It is important that the clinician always remember the drugs are more likely to kill the patient than the disease [19]. 


\section{Conclusion}

Autoimmune dermatologic diseases represent some of the most interesting and challenging problems seen in veterinary dermatology. For instance pemphigus foliaceus (PF) frompemphigus complex and discoid lupus erythematosus (DLE) from lupus erythematosus complex are frequently occurring immune mediated skin diseases. Immunopathogenesis of this group of diseases is the production of antigen-antibody complexes and subsequently lodge in small vessels and the basement membrane zone of the skin and DLE involvement of either abnormal immune regulation or abnormal antigen stimulation to PF is noted.

The risk factors for autoimmune dermatologic diseases are genetics, sex, other immunologic disorders, infectious diseases and drugs. About $60 \%$ of dogs is now purebred and pure breeding always involves inbreeding. This brings genetic complexity which result development and regulation of autoimmune disease. Intact females have the highest incidence of immune diseases and intact males have the lowest. Infections can trigger allergies and the formation of auto antibodies. If autoimmune dermatologic diseases are treated with the appropriate glucocorticoids the prognosis for both of these ailments is fair to good and if not it shifts poor to guard.

\section{Since Recommendations}

i. Since intact females have the highest incidence of immune diseases owners should be informed for spaying of their bitches because ovariohysterectoy reduces the incidence of these ailments.

ii. Whenever the ailment is encountered patients should be treated by appropriate glucocorticoids so that clinician should always remember the drugs are more likely to kill the patient than the disease.

iii. Veterinarians, universities, hospitals and animal wale fare opponents should undertake research on immune mediated dermatologic diseases of dogs in Ethiopia.

iv. The susceptibility to autoimmune diseases is should be controlled by the interplay of environmental and heritable factors so that cross breeding shall be adapted.

\section{References}

1. Garcia KC, Teyton L, Wilson IA (1999) Structural basis of $\mathrm{T}$ cell recognition. Annu Rev Immunol 17: 369-397.
2. Laurel JG (1980) Common Canine Autoimmune Disorders ( ${ }^{\text {rd }}$ edn) National Academic Press Washington, DC, USA, pp. 216-219.

3. Beata U, Katherine B (2011) Major Histocompatibility Complex (MHC) Markers in Conservation Biology. Int J Mol Sci 12(8): 5168-5186.

4. Rimini (2007) Immune Mediated Disease of Small Animals ( $3^{\text {rd }}$ edn), Ludwig Maximilians University, Germany, pp. 143-149.

5. Crawford MA, Foil CS (1989) Vasculitis: Clinical syndromes in small animals. Compend Contin Educ 11: 345-356.

6. Rhea V Morgan, Ronald M Bright, Margaret S Swartout (2003) Hand Book of Small Animal Practice ( $4^{\text {th }}$ edn), Elsevier Science, USA, pp. 894901.

7. Gregory CR (2000) Immunosuppressive agents. In Bonagura JD ( $3^{\text {th }}$ edn), Current Veterinary Therapy XIII, WB Saunders, Philadelphia, pp. 509-513.

8. Ettinger SJ, Feldman EC (2005) Textbook of Veterinary Internal Medicine ( $6^{\text {th }}$ edn), WB Saunders, Philadelphia, pp. 123-129.

9. Campbell RD, Milner CM (1993) MHC genes in autoimmunity. Curr Opin Immunol 5(6): 887-893.

10. Quimby FW, Smith C, Brushwein M, Lewis RW (1980) Efficacy of immunoserodi- agnostic procedures in the recognition of canine immunologic diseases. Amer J Vet Res 41(10): 1662-1666.

11. Day MJ, Penhale WJ (1992) Immune-mediated disease in the old English sheepdog. Res Vet Sci 53(1): 87-92.

12. Adachi K, Yoshimoto A, Hasegawa T, Shimizu T, Goto, Y, et al. (1992) Anti-erythrocyte membrane antibodies detected in sera of dogs naturally infected with Babesiagibsoni. J Vet Med Sci 54(6): 1081-1084.

13. Cribb AE (2008) idiosyncratic reactions to sulfonamides in dogs. Amer Vet Med Ass 195(11): 1612-1614.

14. Michael S (2006) clinical medicine of the dog and cat ( $3^{\text {rd }}$ edn), Manson publishing, London, p. 41-43.

15. Stephen J, Birchard RG, Sherding MS (2006) Saunders Manual of Small Animal Practice ( $3^{\text {rd }}$ edn), An Imprint of Elsevier Inc St Louis, pp. 502509.

16. Scott DW, Miller WH, Griffin CE (2001) Muller and Kirk's Small Animal Dermatology ( $5^{\text {th }}$ edn), WB Saunders Philadelphia, pp. 667-779.

17. Ralf S Mueller, DipACVD, FACVSc (2005) Immune Mediated Skin Diseases ( $3^{\text {rd }}$ edn), Ludwig Maximilians University, Germany, pp. 451 462 .

18. Foil C, Foster AP (2002) Manual of small animal dermatology. British Small Animal Veterinary Associaton, Cheltenham ( $4^{\text {th }}$ edn), pp. 892

19. Thoday KL (2002) Advances in Veterinary Dermatology. Vol 4 Blackwell publishing, Oxford, pp. 789-795.

20. http://emedicine.medscape.com/article

21. http://www.medindia.net/patients/patientsinfo/pemphigus.htm

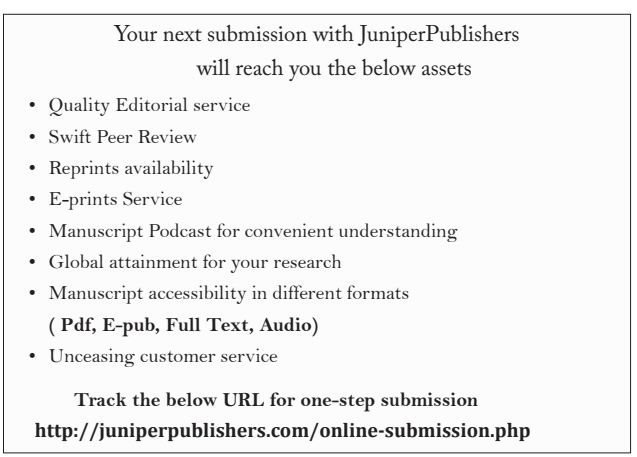

